\title{
Posteroinferior Cerebellar Artery (PICA) Fusiform Aneurysm Ruptured Related with a Pure Arterial Malformation: Case Report and Technical Note (NBCA Embolization under Roadmap-Fluoroscopy)
}

\author{
Maria Del Rosario Sosa-Martinez $(\mathbb{D}$, Guy G. Broc-Haro, Javier Valdes-Garcia, \\ Christopher Mader-Alba, Daniel Juarez-Rebollar
}

Neurosurgery and Neurological Endovascular Therapy Department, “Lic. Adolfo López Mateos” Regional Hospital of the Security and Social Institute of the State Workers, Mexico City, Mexico

Email: rosos24@yahoo.com

How to cite this paper: Sosa-Martine, M. Del R., Broc-Haro, G.G., Valdes-Garcia, J., Mader-Alba, C. and Juarez-Rebollar, D. (2021) Posteroinferior Cerebellar Artery (PICA) Fusiform Aneurysm Ruptured Related with a Pure Arterial Malformation: Case Report and Technical Note (NBCA Embolization under Roadmap-Fluoroscopy). Open Journal of Modern Neurosurgery, 11, 137-143.

https://doi.org/10.4236/ojmn.2021.113017

Received: March 27, 2021

Accepted: May 15, 2021

Published: May 18, 2021

\section{Copyright $\odot 2021$ by author(s) and} Scientific Research Publishing Inc. This work is licensed under the Creative Commons Attribution International License (CC BY 4.0).

http://creativecommons.org/licenses/by/4.0/ (c) (i) Open Access

\begin{abstract}
Background: The pure arterial malformation (PAM) lesion has been recently described as a vascular pathology characterized by the sole presence of coiled, sometimes ectatic, arterial loops. $2 \%$ of the fusiform aneurysms are located in the vertebral-posterior inferior cerebellar arteries. Case Presentation: A 60 years old female with subarachnoid hemorrhage was diagnosed with a fusiform aneurysm in the right PICA related with a PAM in the digital subtraction angiography (DSA). With a negative super-selective Wada test, the patient was treated with embolization of the two lesions and the PICA for being in the same arterial territory with adhesive embolic liquid under roadmap fluoroscopy technique. The patient had a satisfactory clinical evolution and no added neurological deficit, so she was discharged 3 days after the treatment. Conclusion: These two vascular lesions located in the same artery are very rare and more in the PICA territory. It is important to make the best decision to treat them because of the potential complications; that's why the procedure was supported with super-selective Wada test and neuromonitoring of the PICA territory. The injection of the mixture of NBCA and Lipiodol $^{\bullet}$ under roadmap fluoroscopy is very safe.
\end{abstract}

\section{Keywords}

Fusiform Aneurysm, Pure Arterial Malformation, N-Butyl Cyanoacrylate, Roadmap Fluoroscopy 


\section{Background}

The pure arterial malformation (PAM) lesion has been recently described as a vascular pathology characterized by the sole presence of coiled, sometimes ectatic or arterial loops [1]. These lesions are commonly mistaken for aneurysms or arteriovenous malformations [2].

Dolichoectatic arteries and fusiform aneurysms are related to arteriopathies and connective tissue diseases, and just $2 \%$ of the intracranial aneurysms are located in the vertebral-posterior inferior cerebellar arteries (PICAs) [3].

$\mathrm{N}$-butyl cyanoacrylate (NBCA) is commonly used liquid embolic agent in the endovascular treatment of cerebrovascular and peripheral vascular pathologies. Although NBCA is most commonly known as an endovascular treatment option for cerebral arteriovenous malformation, neurointerventionalist has begun to utilize cyanoacrylate glues for several vascular brain pathologies [4] [5].

Our objective is to present two rare types of arterial malformations in the same artery and their successful treatment with endovascular therapy by using a mixture of NBCA and Lipiodol ${ }^{\oplus}$, guided with roadmap fluoroscopy to achieve the proper embolization with good visualization and safe injection.

\section{Case Report}

A 60 years old female, with clinical history of hypertension on enalapril twice daily and no allergies reported. She started 5 days previous admission to the hospital with intensive oppressive headache 10/10 of EVA scale, without neurological focalization and no meningeal signs in the physical examination. The head non-contrast CT scan showed subarachnoid hemorrhage grade IV in the Fisher scale; the blood localization predominantly was in the premedullar and prepontine cisterns and in the IV ventricle (Figure 1). The DSA demonstrated a fusiform aneurysm and a PAM in the second and third segment of the right PICA respectively. The aneurysm configuration was like an arterial dilatation without neck and the PAM had loops, tortuosities and little dilatations (Figure 2). Analyzing the clinical status and the invasive and non-invasive images of the patient radiological chart, we decided to embolize the both lesions as well to occlude the PICA for being in the same arterial territory.

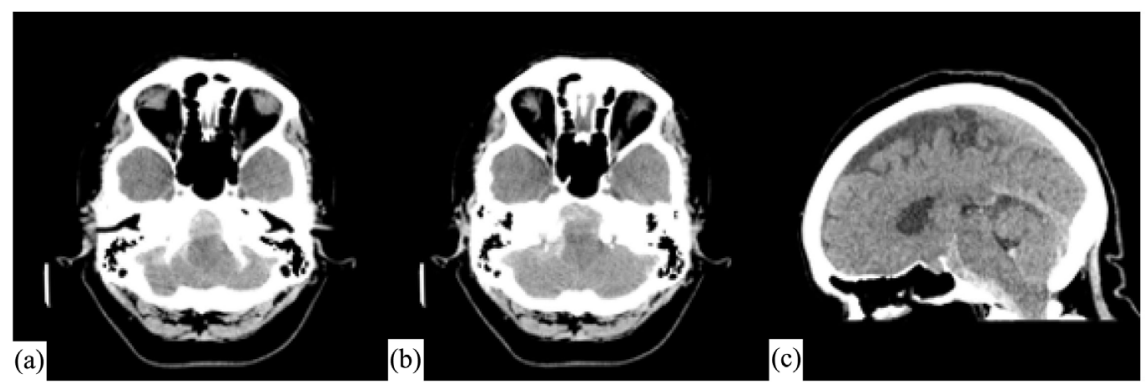

Figure 1. (a), (b) and (c) CT scan demonstrated subarachnoid hemorrhage predominantly in the premedullar, prepontine cisterns and in the IV ventricle. Characterized as a grade IV in the Fisher scale. 

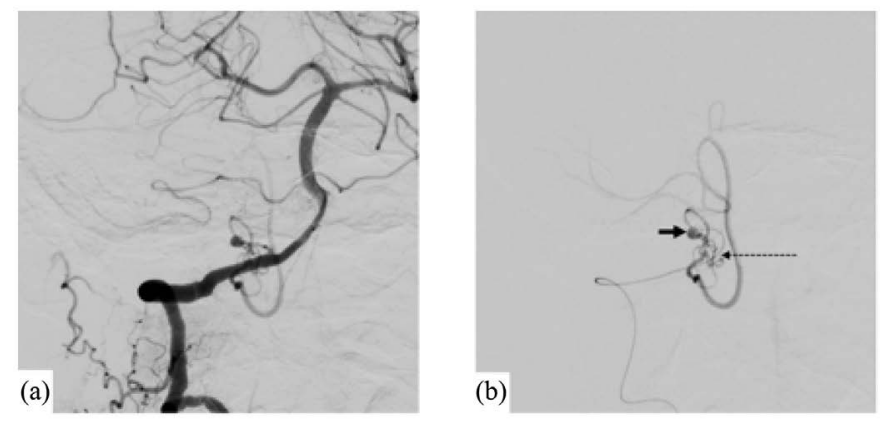

Figure 2. DSA of the right vertebral artery (a) AP Towne projection and (b) Super-selective injection of the right PICA: shows the proximal fusiform aneurysm (second segment) indicated with the black arrow and distal the PAM indicated with the discontinued black arrow (third segment).

The procedure was performed as follows: with the patient awake, analgesia and local anesthesia, neurophysiologic monitoring, super-selective Wada test with 1cc propofol injected in the right PICA obtaining as result, no neurophysiological deficit and clinically did not develop a Wallenberg syndrome.

We proceeded to embolize both lesions and the right PICA with NBCA and Lipiodol $^{\circ}$. The patient returned to the ICU and she had a satisfactory clinical evolution and no added neurological deficit, so she was discharged 3 days after the treatment. In the follow up as outpatient 5 months after the procedure she had a grade $2 \mathrm{mRS}$.

\section{Management}

We approached the right femoral artery with a $6 \mathrm{~F}$ sheath. For the right vertebral hypoplastic artery was used a $5 \mathrm{~F}$ Chaperon ${ }^{\circledR}$ for the coaxial system with a 1.5 Headway Duo $^{\circ}$ microcatheter and a Xpedion ${ }^{\mathrm{TM}} 10$ microwire for the super-selective catheterization of the right PICA under roadmap fluoroscopy and done a super-selective angiogram (Figure 2(a)) provocative Wada test was performed with $1 \mathrm{cc}$ of propofol injected in the right PICA supported with neuromonitoring and physical exploration of the patient. The result of the test was negative for a neurological deficit. A load dose of dexamethasone was given intravenously, the microcatheter in optimal position was flushed with $10 \mathrm{cc}$ of $5 \%$ glucose solution, the embolization was done with a dilution 1:8 of NBCA and Lipiodol $^{\circledR}$ under roadmap fluoroscopy technique in a Philips Allura. In Figure 3: we see the microcatheter tip in the second segment of the PICA and the beginning of the embolization as well as the radiopacity, of the NBCA + Lipiodol $^{\varpi}$ with this technique and finally the detached of the microcatheter.

In the ending rounds of the DSA showed total exclusion of the aneurysm, PAM and the right PICA occlusion (Figure 4(a)); the parenchymogram demonstrated no vascular defects (Figure 4(b)).

The femoral artery was closed with Angioseal ${ }^{\oplus} 6 \mathrm{~F}$ and the control non contrast CT scan did not demonstrate hemorrhage or ischemic stroke, the liquid embolic material with no artifact (Figure 5). 


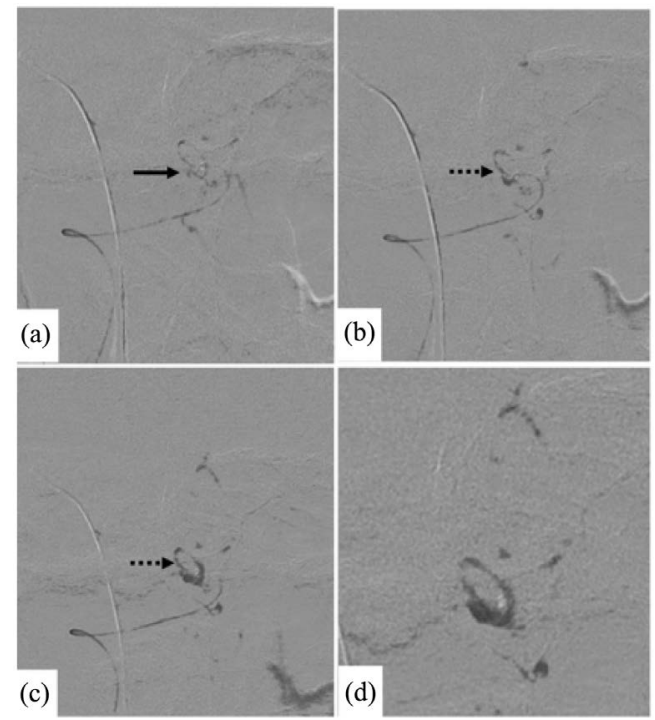

Figure 3. The NBCA diluted with Lipiodol $^{\circledR}$ was injected under roadmap fluoroscopy technique. (a) We see the microcatheter tip and the beginning of the embolization with the adhesive liquid mixture (black arrow). (b) and (c) We demonstrate the radiopacity, advanced and penetration of the NBCA + Lipiodol ${ }^{\oplus}$ (black discontinued arrow). (d) The last round roadmap fluoroscopy shows the detached microcatheter.
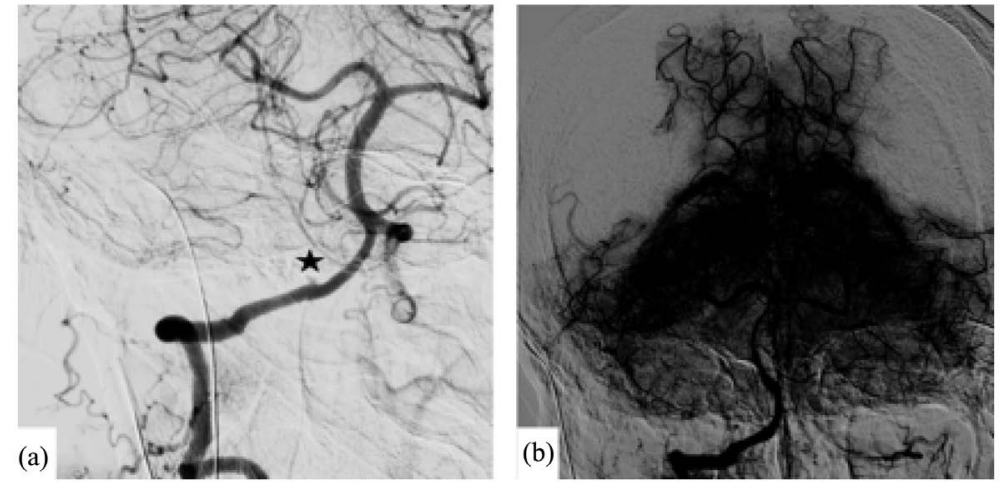

Figure 4. Postoperative control DSA. (a) The aneurysm, pure arterial malformation and the PICA are excluded (black star) from the arterial circulation. (b) No vascular zone defects in the caudal portion of the cerebellum in the parenchymogram of the arterial territory treated.
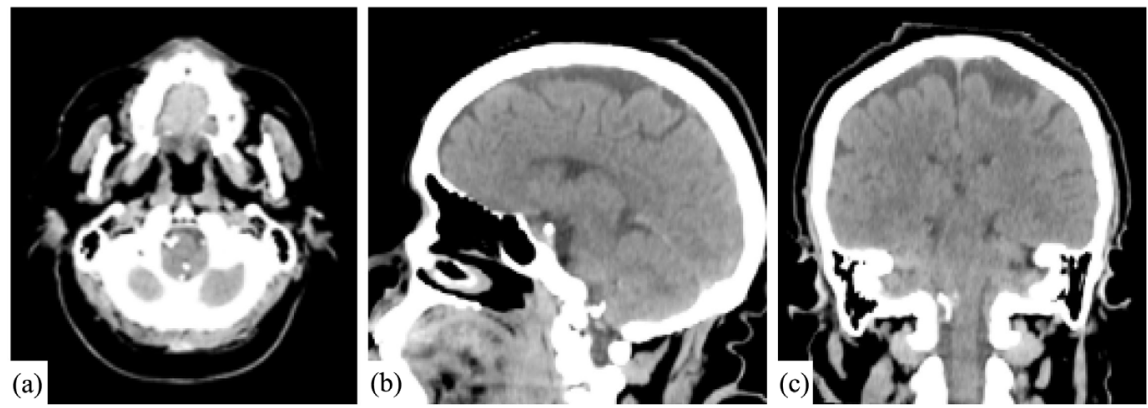

Figure 5. Noncontrast head CT scan (c) Axial, (d) Sagittal and (c) Coronal projection revealed the radiopacity and no artifact generated by the liquid embolic material in the PICA territory. In all imagens there is no evidence of hemorrhage or ischemic stroke. 


\section{Discussion}

In pure arterial malformations the overlapping, dilated and tortuous trajectory are coil-like appearance in all population ages and may have genetic, infectious, inflammatory, immunological o degenerative predisposing factors that may cause or contribute to the formation and/or progression of the arteriopathy. The appropriate characterization of the vasculopathy architecture is paramount important for choosing the best treatment and prognosis varies depending on the type of the vascular anomalies [6].

Aneurysms arising from the PICA distal to the origin from the vertebral artery are rare, represent $0.5 \%$ - $3 \%$ of all intracranial aneurysms. The fusiform geometry is considered when they had no discernible neck [7]. And PICA aneurysms are most commonly discovered when patients present a subarachnoid hemorrhage (SAH), although patients can occasionally present symptoms related to the medulla and cranial nerves IX - XI. These lesions can be treated by surgery and have several advantages, as immediate decompression by removing the blood clot and identification of the bleeding sources in cases with multiple lesions and decrease the risk of re-ruptured [8] [9]. Approximately $20 \%$ to $66 \%$ of the lower cranial nerves deficits are associated with open surgery. However, several studies have been published stating the usefulness of endovascular management that avoids the manipulation of the posterior fossa structures [9].

In the current clinical case, the CT scan and the DSA led us to conclude that the Fisher Grade IV subarachnoid hemorrhage, was secondary to the rupture of the fusiform aneurysm because of the high hemodynamic shear stress of the aneurysmal wall occasioned by the PAM.

To evaluate a possible infarction of the PICA territory, we used super-selective provocative test with propofol, this anesthetic has a safety margin, low incidence of side effects, and effectiveness in inducing controlled transitory losses of neurologic function in the perfused areas [10] [11].

The embolization was done with previous IV load dose of dexamethasone and the microcatheter flushed with $10 \mathrm{cc}$ of $5 \%$ glucose. With N-butyl cyanoacrylate (NBCA) and Lipiodol ${ }^{\circledR}$ with a dilution 1:8, under roadmap fluoroscopy technique; the injection of the glue mixture thru the right PICA was for 65 seconds (Figure 4). We showed the good visualization, advance and penetration of the embolic liquid mixture during the injection and excellent control of the reflux at the tip of the microcatheter; this is important to accomplish the goal of the procedure as well as to choose the right moment for the withdrawal of the microcatheter.

The iodine component of the Lipiodol $^{\circledR}$ determines it is the radiopacity so during the injection of this embolic material under roadmap fluoroscopy technique and makes unnecessary the addition of tantalum or tungsten for the proper control and visualization during the injection as when using fluoroscopy only.

Lipiodol ${ }^{\circledR}$ has 2 functions: delays the polymerization of the NBCA by shielding it from surrounding ionic substances and the radiopacity during the injection 
under roadmap fluoroscopy.

The glue composition depends on the hemodynamic characteristics of the targeted lesion to calculate the viscosity of the embolic [12] and the radiopacity adding tantalum or tungsten powder to increase the visibility of the mixture in the conventional fluoroscopy technique [13] [14] [15].

\section{Conclusions}

There is a low incidence of a fusiform and a pure arterial malformation in the same intracranial arterial territory. The endovascular technique is a secure procedure, because during the treatment you can do neurological provocative test and decide the most suitable management.

The utilization of the roadmap fluoroscopy technique during the injection of the mixture of NBCA and Lipiodol ${ }^{\circ}$, gives the operator the visibility of the radiopacity, advance and penetration of the glue as well the reflux at the microcatheter tip without adding tantalum and tungsten.

\section{Acknowledgements}

Jorge L. Balderrama Bañares MD. Chief of the Neurological Endovascular Therapy Department of the Neurology and Neurosurgery National Institute. Mexico City.

For his advices for management of complex clinical cases.

\section{Conflicts of Interest}

The authors have no ethical conflicts.

\section{References}

[1] Sorenson, T.J., Brinjikji, W., Flemming, K.D. and Lanzino, G. (2018) Pure Arterial Malformation of the Posterior Inferior Cerebellar Artery with Interspersed Adiposen Tissue: Case Report. Journal of Neurosurgery: Pediatrics PED, 22, 261-264. https://doi.org/10.3171/2018.4.PEDS18135

[2] Brinjikji, W., Cloft, H.J., Flemming, K.D., Comelli, S. and Lanzino, G. (2018) Pure Arterial Malformations. Journal of Neurosurgery, 129, 91-99. https://doi.org/10.3171/2017.2.JNS1744

[3] Steiger, J.H., Etmainan, N. and Hanggi, D. (2015) Chapter 2: Pathophysiology and Anatomy. In: Microsurgical Brain Aneurysms, Springer, Berlin, Heidelberg, 7-25. https://doi.org/10.1007/978-3-662-45679-8 2

[4] Cuoco, J.A., Do, M.S., Guilliams, E.L., Kelin, B.J., Malaty, G.R., Witcher, M.R., et al. (2020) N-Butyl Cyanoacrylate Embolization of a Traumatic Pseudoaneurysm and Aretriasvenous Fistula of the Middle Meningeal Artery. Radiology Cases Report, 15, 321-325. https://doi.org/10.1016/j.radcr.2019.12.009

[5] Kim, D., Lee, J.Y., Jeon, H.J., Cho, B.M., Park, S.H. and Oh, S.M. (2015) Intraoperative Endovascular Embolization of Middle Memingeal Artery and Pseudoaneurysm by Using N Butyl 2 Cyanoacrylate for Hemostasis during Operation of Acute Epidural Hemorrhage. Korean Journal of Neurotrauma, 11, 167-169. https://doi.org/10.13004/kjnt.2015.11.2.167 
[6] McLaughlin, N., Raychev, R., Duckwiler, G. and Martin, N.A. (2013) Pure Arterial Malformation of the Posterior Cerebral Artery: Importance of Its Recognition. Journal of Neurosurgery, 119, 655-660. https://doi.org/10.3171/2013.4.JNS121374

[7] Nussbaum, E.S., Mendez, A., Camarata, P. and Sebring, L. (2003) Surgical Management of Fusiform Aneurysms of the Peripheral Posteroinferior Cerebellar Artery. Neurosurgery, 53, 831-835. https://doi.org/10.1227/01.NEU.0000084162.29616.43

[8] Lewis, S.B., Chang, D.J., Peace, D.A., La Frentz, P.J. and Day, A.L. (2002) Distal Posterior Inferior Cerebellar Artery Aneurysms: Clinical Features and Management. Journal of Neurosurgery, 97, 756-766. https://doi.org/10.3171/jns.2002.97.4.0756

[9] Xu, F., Hong, Y., Zheng, Y., Xu, Q. and Leng, B. (2017) Endovascular Treatment pf Posterior Inferior Cerebellar Artery Aneurysms: A 7-Year Single-Center Experience. Journal of NeuroInterventional Surgery, 9, 45-51.

https://doi.org/10.1136/neurintsurg-2016-012489

[10] Feliciano, C.E., De León-Berra, R., Hernández Gaitán, M.S., Torres, H.M., Creagh, O. and Rodríguez-Mercado, R. (2010) Provocative Test with Propofol: Experience in Patients with Cerebral Arteriovenous Malformations who Underwent Neuroendovascular Procedures. American Journal of Neuroradiology, 31, 470-475. https://doi.org/10.3174/ajnr.A1855

[11] Curot, J., Denuelle, M., Busigny, T., Barragan-Jason, G., Kany, M., Tall, P., Marlat, F., Fabre, N. and Valton, L. (2014) Bilateral Wada Test: Amobarbital or Propofol? Seizure, 23, 122-128. https://doi.org/10.1016/j.seizure.2013.10.009

[12] Gonzalez, L.F., Albuquerque, F.C. and McDougall, C. (2015) Chapter 30: Tricks of the Trade. In: Fernando, G.L., Albuquerque, F.C. and McDougall, C.G., Eds., Neurointerventional Techniques, Thieme, New York, 154-157. https://doi.org/10.1055/b-0034-102504

[13] The n-BCA Trial Investigators (2002) N-Butyl Cyanoacrylate Embolization of Cerebral Arteriovenous Malformations: Results of a Prospective, Randomized, Multi-Center Trial. American Journal of Neuroradiology, 23, 748-755.

[14] Tamatani, S., Koike, T., Ito, Y. and Tanka, R. (2000) Embolization of Arteriovenous Malformation with Diluted Mixture of NBCA. Interventional Neuroradiology, 6, 187-190. https://doi.org/10.1177\%2F15910199000060S129

[15] Takeuchi, Y., Morishita, H., Sato, Y., Hamaguchi, S., Hamaguchi, S., Sakamoto, N., Tokue, H., et al. (2012) Guidelines for the Use of NBCA in Vascular Embolization Devised by the Committee of Practice Guidelines of the Japanese Society of Interventional Radiology (CGJSIR), 2012 Edition. Japanese Journal of Radiology, 32, 500-517. https://doi.org/10.1007/s11604-014-0328-7 